\title{
Isolation and Identification of Rhizopus oligosporus Local Isolate Derived from Several Inoculum Sources
}

\author{
A. S. Duniaji ${ }^{1 *}$, W. Wisaniyasa ${ }^{2}$, N. N. Puspawati ${ }^{3}$ and dan N. M. Indri $\mathbf{H}^{4}$ \\ Department of Food Science and Technology Udayana University, Bali, Indonesia \\ *Corresponding author
}

\section{A B S T R A C T}

\section{Keywords Identification, $R$. oligosporus, local isolate, Inoculums. \\ Article Info \\ Accepted: \\ 12 August 2019 \\ Available Online: \\ 10 September 2019}

This study was aims to obtain local isolates of Rhizopus oligosporus from several sources of inoculums such as Herbicus leaves, Tectona leaves, various brands of tempeh and starter of tempeh. Rhizopus oligosporus is a fungus that belongs to the Zygomycetes group, which is one of two classes in the Zygomycota phylum. Role of $R$. oligosporus in the popular Indonesian food Tempe fermentation. A total of 72 samples were isolated from various sources of inoculum consisting of 18 samples from waru leaves, 18 samples from teak leaves, 18 samples from various brands and the origin of tempe and 18 samples from various brands and origin of tempeh starter. The method used in this study was to isolate and identify $R$. oligosporus local isolates from waru leaves, teak leaves various brands of tempe and tempeh starter using Potato Dextrose Agar (PDA), Malt Extract Agar (MEA), Czapek Yeast Extract Agar (CYA). The results showed that mold types $R$. oryzae, $R$. oligosporus and other molds with populations ranged from $1.5 \times 10^{2} \mathrm{cfu} / \mathrm{g}-9.5 \times 10^{2} \mathrm{cfu} / \mathrm{g}$, $0.5 \times 10^{3}-9.6 \times 10^{3} \mathrm{cfu} / \mathrm{g}$ and $1.6 \times 10^{3} \mathrm{cfu} / \mathrm{g}-8.6 \times 10^{2} \mathrm{cfu} / \mathrm{g}$. A total of 72 samples taken from various sources of inoculums, $R$. oligosporus found as many as 12 from Herbicus leaves, 3 samples from Tectona leaves, 17 samples from various brands of tempeh and as many as 11 samples from tempeh starter were identified macroscopically based on color conidia with brownish gray color and the growth of mycelia and conidia is very dense. Macroscopically and microscopically identified as many as 19 local isolates of $R$. oligosporus which have the potential as selected fungi in food processing.

\section{Introduction}

$R$. oligosporus is a fungus of the family Mucoraceae and is a widely used starter culture for the production of tempeh at home and industrially. As the mold grows it produces fluffy, white mycelia, binding the beans together to create an edible "cake" of partly catabolized soybeans. The domestication of the microbe is thought to have occurred in Indonesia several centuries ago (Shurtleff and Aoyagi, 2001, A. 2001; Abe et al., 2010; Jennessen et al., 2008)

$R$. oligosporus is the preferred starter culture for tempeh production for several reasons. It grows effectively at high temperatures (30$40{ }^{\circ} \mathrm{C}$ ) which are typical of the Indonesian islands, it exhibits strong lipolytic and proteolytic activity that create desirable 
properties in tempeh and it produces metabolites that allows it to inhibit and thus outcompete other molds and gram-positive bacteria, including the potentially harmful Aspergillus flavus and Staphylococcus aureus (Jennessen et al., 2008; Hessel Tine et al., 1940; Shurtleff and Aoyagi, 2001). $R$. oligosporus is at present considered to be a domesticated form of Rhizopus microsporus and its proper taxonomic position is thus Rhizopus microsporus var. oligosporus.

R. microsporus produces several potentially toxic metabolites, rhizoxin and rhizonins A and $\mathrm{B}$, but it appears the domestication and mutation of the R. oligosporus genome has led to the loss of genetic material responsible for toxin production (Fardiaz 1989; Jennessen et al., 2005; Madigan and Martinko, 2006)

Although other varieties in Rhizopus microscopus may be harmful, $R$. oligosporus is not associated with production of potentially harmful metabolites. It is not found in nature and is frequently used by humans (Pitt and Hocking,. 1985; Samson et al., 2005; Dewi and Aziz, 2011) Rhizopus oligosporus strains have a large (up to $43 \mathrm{~mm}$ ) and irregular spores with the most variable sizes.

This is, for instance, reflected as high values in the spore volume (96-223 mm3/spore). $R$. oligosporus has large, subglobose to globose spores, and high proportion irregular spores (>10\%). R. oligosporusalso has spores with nonparallel valleys and ridges, and plateaus that sometimes are granular (Pitt and Hocking, 1985; Samson et al., 2005). R. oligosporus Saito has brownish gray colonies with a height of $1 \mathrm{~mm}$ or more. Single sporangiofor or in groups with smooth or slightly rough walls, with a length of more than $1000 \mathrm{um}$ and a diameter of 10-18 um. Sporangia globosa, which is brownish-black in color, with a diameter of 100-180 um (Pitt and Hocking, 1985; Samson et al., 2005; Jennessen et al.,
2008). The research was aimed to isolation and identificatin of $R$. oligosporus derived from several leaf of plant and inoculums of tempeh and their starter. On the other hand $R$. oligosporus is used to inhibit Aspergillus parasiticus that is a patogen fungi harmfull of the heathy of human and animal. Aspergillus parasiticus was also produce secundary metabolite toxic aflatoxin B1.

\section{Materials and Methods}

This study was conducted from March 2018 to November 2018 in the Food Microbiology laboratory of the Food Science and Technology Study Program, Faculty of Agricultural Technology and Laboratory of Pest and disease of Plant, Faculty of Agriculture of Udayana University.

Sampling of Herbicus leaves, Tectona leaves, various brands and the origin of tempeh and various brands and origin of tempeh yeast in several provinces of Bali.

\section{Rhizopus oligosporus Isolate}

Local isolates of $R$. oligosporus in this study were obtained from isolation from various sources of inoculums, namely Herbicus leaves, Tectona leaves, various brands and origin of tempe and various brands and origin of tempeh starter in several provinces of Bali. Maintenance of isolates through regeneration every 3 months by inoculating on oblique Potato Dextrose agar (PDA) media and incubated $30 \mathrm{oC}$ for 7 days. Furthermore, it is stored in refrigerator $4 \mathrm{o} \mathrm{C}$.

\section{Media isolation, purification, storage and maintenance of isolates}

Media for growing molds using Potato Dextrose agar (PDA) agar media. Each 1000 $\mathrm{ml}$ medium was made from $200 \mathrm{~g}$ of potatoes, $20 \mathrm{~g}$ of glucose, $20 \mathrm{~g}$ of agar, $0.1 \mathrm{~g}$ of 
chloramphenicol and sterilized at 121o $\mathrm{C}$ for 15 minutes. The obtained fungal isolates were observed macroscopically and microscopically

\section{Media identification (Pitt and Hocking,} 1985)

Isolates on medium of Potato Dextrose agar (PDA) were then transferred to Malt Extract Agar (MEA) media, Czapek Yeast Extract Agar (CYA), 25\% Glycerol Nitrate Agar (G25N) for identification. The composition of Malt Extract Agar (MEA) media per $1000 \mathrm{ml}$ is $20 \mathrm{~g}$ of malt extract powder, $1.0 \mathrm{~g}$ of peptone, $20 \mathrm{~g}$ of glucose, $20 \mathrm{~g}$ of agar. The three media above were sterilized 121 o $\mathrm{C}$ for 15 minutes. The composition of Czapek Yeast Extract Agar (CYA) per $1000 \mathrm{ml}$ is $1.0 \mathrm{~g}$ K2HPO4, $10 \mathrm{ml}$ czapek concentrate, $5.0 \mathrm{~g}$ yeast extract powder, $30 \mathrm{~g}$ sucrose, $15 \mathrm{~g}$ agar. While the composition of Czapek Concentrate in $100 \mathrm{ml}$ is $30 \mathrm{~g} \mathrm{NaNO} 3,5.0 \mathrm{~g} \mathrm{KCl}, 5.0 \mathrm{~g}$ $\mathrm{MgSO} 47 \mathrm{H} 2 \mathrm{O}, 0.1 \mathrm{~g}$ FeSO4.7H2O. Media composition of $25 \%$ Glycerol Nitrate Agar $(\mathrm{G} 25 \mathrm{~N})$ per $1000 \mathrm{ml}$ is $0.75 \mathrm{~g} \mathrm{~K} 2 \mathrm{HPO} 4,7.5$ ml Czapek concentrate, $3.7 \mathrm{~g}$ yeast extract powder, $250 \mathrm{~g}$ glycerol, $12 \mathrm{~g}$ agar.

\section{Isolation and purification of Rhizopus spp}

The stages of isolation and purification of Rhizopus spp were carried out by taking aseptically the dominant isolate from tempeh which was inoculated on Potato Dextrose Agar (PDA) media and incubated at $30^{\circ} \mathrm{C}$ for 24 hours until a colony formed. Transfer of isolate (purification) is repeated 4 times until the isolate is completely pure. Flowchart of isolation and purification stages can be seen in Figure 1 (modified Pitt and Hocking, 1985)

\section{Identification $\boldsymbol{R}$. oligosporus}

Mushroom isolates on oblique Potato Dextrose Agar (PDA) media are regrouped according to the origin of the inoculums.
Identification was limited to isolates with brownish grayish conidia color using Czapek Yeast Extract Agar (CYA) media, 25\% Glycerol Nitrate Agar (G25N), Malt Extract Agar (MEA) incubated for 7 days at $5^{\circ} \mathrm{C}, 25$ $\circ \mathrm{C}$, and $37^{\circ} \mathrm{C}$ (modified Pitt and Hocking, 1985).

\section{Results and Discussion}

\section{Population of Rhizopus spp.}

The result of mold isolation from various sources of inoculums showed that from 72 samples studied found variations in mold types based on colony color and morphology both macroscopically and microscopically. In Table 1, the isolated fungi population from various sources of inoculums were shown.

$R$. oligosporus is a mold from the phylum Zygomycota which is able to produce protease enzymes. $R$. oligosporus is commonly found in rotting soil, fruits and vegetables, and old bread. $R$. oligosporus is included in Zygomycota which is often used in making tempeh from the fermentation process of soybeans, because $R$. oligosporus which produces phytase enzymes that break down phytate makes macro components in soybeans broken down into micro components so that tempeh is more easily digested and nutrients are more easily absorbed by the body. This fungus can also ferment other substrates, produce enzymes, and treat waste. One of the enzymes produced is from the protease group. (Rahayu, K. 1988; Abe et al., 2010; Jennessen et al., 2008)

$R$. oligosporus is a fungus of the family Mucoraceae and is a widely used starter culture for the production of tempeh at home and industrially. As the mold grows it produces fluffy, white mycelia, binding the beans together to create an edible "cake" of partly catabolized soybeans. The 
domestication of the microbe is thought to have occurred in Indonesia several centuries ago. (Hessel Tine et al., 1940; Shurtleff and Aoyagi, 2001; Abe et al., 2010; Jennessen et al., 2006). In Table 2. Can be seen characteristic of rhizopus spp from herbicus leaves in macroscopically

$R$. oligosporus has brownish gray colonies with a height of $1 \mathrm{~mm}$ or more. A single person or group in groups with smooth or rather rough walls, with a length of more than 1000 micro meters and a diameter of 10-18 micro meters. Sporangia globosa is brownish black, with a diameter of 100-180 micro meters. Chlamydospores are many, single or short, colorless chains, containing granules, formed on hyphae, sporangiophores and sporangia. The form of Chlamidospora globosa, ellipse or cylindrical with a size of 730 micro meters or 12-45 micro meters x 7-35 micro meters (Pitt and Hocking, 1985; Samson et al., 2005; Jennessen et al., 2008)

$R$. oligosporus can grow optimally at a temperature of $30-35^{\circ} \mathrm{C}$, with a minimum temperature of $12^{\circ} \mathrm{C}$, and a maximum temperature of $42^{\circ} \mathrm{C}$. Some of the benefits of $R$. oligosporus include its enzymatic activity, ability to produce natural antibiotics that specifically fight gram-positive bacteria, biosynthesis of vitamins $\mathrm{B}$, its need for carbon and nitrogen source compounds, spore germination, and penetration of tempeh mycelia fungus into tissues soybean seeds (Kobayasi et al.,1992; Pitt and Hocking, 1985; Samson et al., 2005; Jennessen et al., 2008). In Table 3 can be seen characteristic of rhizopus spp from tectona leaves in macroscopically

R. oligosporus is the preferred starter culture for tempeh production for several reasons. It grows effectively at high temperatures (30$40^{\circ} \mathrm{C}$ ) which are typical of the Indonesian islands, it exhibits strong lipolytic and proteolytic activity that create desirable properties in tempeh and it produces metabolites that allows it to inhibit and thus outcompete other molds and gram-positive bacteria, including the potentially harmful Aspergillus flavus and Staphylococcus aureus (Kobayasi et al.,1992; Rahayu, K. 1988; Shurtleff and Aoyagi, 2001; Madigan and Martinko, 2006). Table 4 can be seen characteristic of rhizopus spp from tempeh macroscopically $R$. oligosporus is at present considered to be a domesticated form of $R$. microsporus and its proper taxonomic position is thus $R$. microsporus var. oligosporus. $R$. microsporus produces several potentially toxic metabolites, rhizoxin and rhizonins $\mathrm{A}$ and $\mathrm{B}$, but it appears the domestication and mutation of the $R$. oligosporus genome has led to the loss of genetic material responsible for toxin production (Jennessen et al., 2006; Samson et al., 2005; Jennessen et al., 2008). Table 5. Can be seen characteristics of rhizopus spp from starter of tempeh macroscopically

Macroscopically and microscopically isolates of Rhizopus spp with brownish gray color are presented in Figures 1, 2 and 3.

To support species determination or to evaluate the possibilities of adopting infraspecific classification of Zheng et al., (2007) for those 35 strains in $R$. microsporus, morphological and physiological characteristics were then observed.

Morphological characteristics, such as sporangiophore (length and colour), columellae (shape), sporangiospore (shape, size, and colour), and rhizoidetype were examined according to Zheng et al., (2007) by using light microscope Olympus ${ }^{\text {TMBX53 }}$ (Olympus, Japan). Measurements of sporangiophore length and sporangiospore size were made in 30 replications (n 1/4 30). 
Table.1 Population of molds from several of inoculums

\begin{tabular}{|l|c|c|c|}
\hline Inoculom sources & $\boldsymbol{R}$. oryzae $(\mathbf{C f u} / \mathbf{g})$ & $\begin{array}{c}\boldsymbol{R} \text {. oligosporus } \\
\text { (Cfu/g) }\end{array}$ & Other molds $(\mathbf{C f u} / \mathbf{g})$ \\
\hline Herbicus leaves 1 & $5.0 \times 10^{2}$ & $6.3 \times 10^{3}$ & $1.7 \times 10^{2}$ \\
\hline Herbicus leaves 2 & $5.2 \times 10^{2}$ & $8.2 \times 10^{3}$ & $2.8 \times 10^{2}$ \\
\hline Herbicus leaves 3 & $5.6 \times 10^{2}$ & $9.1 \times 10^{3}$ & $1.6 \times 10^{2}$ \\
\hline Tectona leaves 1 & $8.4 \times 10^{2}$ & $0.5 \times 10^{2}$ & $8.6 \times 10^{2}$ \\
\hline Tectona leaves 2 & $7.6 \times 10^{2}$ & $1.0 \times 10^{2}$ & $7.9 \times 10^{2}$ \\
\hline Tectona leaves 3 & $9.5 \times 10^{2}$ & $1.2 \times 10^{2}$ & $6.6 \times 10^{2}$ \\
\hline Tempeh 1 & 0 & $7.2 \times 10^{3}$ & 0 \\
\hline Tempeh 2 & 0 & $9.6 \times 10^{3}$ & 0 \\
\hline Tempeh 3 & 0 & $8,0 \times 10^{3}$ & 0 \\
\hline Starter of Tempeh 1 & $2.5 \times 10^{2}$ & $9,5 \times 10^{3}$ & 0 \\
\hline Starter of Tempeh 2 & $2.2 \times 10^{2}$ & $6,5 \times 10^{3}$ & 0 \\
\hline Starter of Tempeh & $1.5 \times 10^{2}$ & $4,5 \times 10^{3}$ & 0 \\
\hline
\end{tabular}

Table.2 Characteristic of Rhizopus spp from Herbicus leaves in macroscopically

\begin{tabular}{|c|c|c|c|c|c|}
\hline \multirow[t]{2}{*}{ No } & \multirow[t]{2}{*}{ Code of Isolate } & \multirow[t]{2}{*}{$\begin{array}{ll}\text { Sources } & \text { of } \\
\text { Inoculums }\end{array}$} & \multirow[t]{2}{*}{$\begin{array}{l}\text { Color of } \\
\text { Colony }\end{array}$} & \multicolumn{2}{|c|}{$\begin{array}{c}\text { Observation in medium } \\
\text { agar }\end{array}$} \\
\hline & & & & Mycelia & Conidia \\
\hline 1 & WS1 & Sanur & Brownish Gray & +++ & +++ \\
\hline 2 & WS2 & Sanur & Brownish Gray & +++ & +++ \\
\hline 3 & WS3 & Sanur & Brownish Gray & +++ & +++ \\
\hline 4 & WR1 & Renon & Brownish Gray & +++ & +++ \\
\hline 5 & WR2 & Renon & Brownish Gray & +++ & +++ \\
\hline 6 & WR3 & Renon & Brownish Gray & +++ & +++ \\
\hline 7 & WP1 & Padanggalak & gray & ++ & ++ \\
\hline 8 & WP2 & Padanggalak & gray & ++ & ++ \\
\hline 9 & WP3 & Padanggalak & gray & ++ & ++ \\
\hline 10 & WK1 & Kesiman & Brownish Gray & +++ & +++ \\
\hline 11 & WK2 & Kesiman & Brownish Gray & +++ & +++ \\
\hline 12 & WK3 & Kesiman & Brownish Gray & +++ & +++ \\
\hline 13 & WJ1 & Jimbaran & gray & +++ & +++ \\
\hline 14 & WJ2 & Jimbaran & gray & ++ & +++ \\
\hline 15 & WJ2 & Jimbaran & gray & ++ & +++ \\
\hline 16 & WB1 & Bukit & Brownish Gray & +++ & +++ \\
\hline 17 & WB2 & Bukit & Brownish Gray & +++ & ++ \\
\hline 18 & WB3 & Bukit & Brownish Gray & +++ & ++ \\
\hline
\end{tabular}

Remarks : +++ : Very Thick (cover all over PDA sloped), ++ : Thick (Cover 3/4 PDA sloped on 5 days) 
Table.3 Characteristic of Rhizopus spp from Tectona leaves in macroscopically

\begin{tabular}{|c|c|c|c|c|c|}
\hline \multirow[t]{2}{*}{ S.No. } & \multirow[t]{2}{*}{ Code Isolate } & \multirow{2}{*}{$\begin{array}{l}\text { Sources } \\
\text { Inoculums }\end{array}$} & \multirow{2}{*}{$\begin{array}{l}\text { Color of } \\
\text { Colony }\end{array}$} & \multicolumn{2}{|c|}{ Observation in medium agar } \\
\hline & & & & Miselia & konidia \\
\hline 1 & JJ1 & Jimbaran & Gray & +++ & ++ \\
\hline 2 & $\mathrm{JJ} 2$ & Jimbaran & Gray & +++ & ++ \\
\hline 3 & $\mathrm{JJ} 3$ & Jimbaran & Gray & ++ & ++ \\
\hline 4 & JF1 & Kampus FTP & Gray & +++ & +++ \\
\hline 5 & JF2 & Kampus FTP & Gray & +++ & +++ \\
\hline 6 & JF3 & Kampus FTP & Gray & ++ & ++ \\
\hline 7 & JT1 & Kampus Teknik & Brownish Gray & +++ & +++ \\
\hline 8 & JT2 & Kampus Teknik & Brownish Gray & +++ & +++ \\
\hline 9 & JT3 & Kampus Teknik & Gray & +++ & ++ \\
\hline 10 & JM1 & Kampus MIPA & Gray & +++ & +++ \\
\hline 11 & JM1 & Kampus MIPA & Gray & +++ & +++ \\
\hline 12 & JM1 & Kampus MIPA & Gray & ++ & ++ \\
\hline 13 & JS1 & Singaraja & Brownish Gray & ++ & +++ \\
\hline 14 & JS2 & Sigaraja & Gray & ++ & ++ \\
\hline 15 & JS3 & Singaraja & Gray & +++ & +++ \\
\hline 16 & JK1 & Denpasar & Gray & ++ & +++ \\
\hline 17 & JK2 & Denpasar & Gray & ++ & ++ \\
\hline 18 & JK3 & Denpasar & Gray & +++ & +++ \\
\hline
\end{tabular}

Remarks : +++ : Very Thick (cover all over PDA sloped ), ++ : Thick (Cover 3/4 PDA sloped on 5 days)

Table.4 Characteristic of Rhizopus spp from Tempeh Macroscopically

\begin{tabular}{|c|c|c|c|c|c|}
\hline \multirow[t]{2}{*}{ No } & \multirow[t]{2}{*}{ Code Isolate } & \multirow[t]{2}{*}{ Sources of Inoculums } & \multirow{2}{*}{$\begin{array}{l}\text { Color of } \\
\text { Conidia }\end{array}$} & \multicolumn{2}{|c|}{ Observation in Medium Agar } \\
\hline & & & & Miselia & konidia \\
\hline 1 & TT1 & None Merk & Brownish Gray & +++ & +++ \\
\hline 2 & TT2 & None Merk & Brownish Gray & +++ & +++ \\
\hline 3 & TT3 & None Merk & Brownish Gray & +++ & +++ \\
\hline 4 & TL1 & Merk Langgeng & Brownish Gray & +++ & +++ \\
\hline 5 & TL2 & Merk Langgeng & Brownish Gray & +++ & +++ \\
\hline 6 & TA1 & Merk Arya & Brownish Gray & +++ & +++ \\
\hline 7 & TA2 & Merk Arya & Brownish Gray & +++ & +++ \\
\hline 8 & TF1 & Merk Family & Brownish Gray & +++ & +++ \\
\hline 9 & TF2 & Merk Family & Brownish Gray & +++ & +++ \\
\hline 10 & TM1 & Merk Murni & Brownish Gray & +++ & +++ \\
\hline 11 & TM2 & Merk Murni & Brownish Gray & +++ & +++ \\
\hline 12 & TP1 & None Merk & Brownish Gray & +++ & +++ \\
\hline 13 & TP2 & None Merk & Brownish Gray & +++ & +++ \\
\hline 14 & $\mathrm{TC} 1$ & Merk Cak & Brownish Gray & +++ & +++ \\
\hline 15 & $\mathrm{TC} 2$ & Merk Cak & Brownish Gray & +++ & +++ \\
\hline 16 & TG1 & None Merk & Brownish Gray & +++ & +++ \\
\hline 17 & TG2 & None Merk & Brownish Gray & +++ & +++ \\
\hline 18 & TS1 & None Merk (Singaraja) & Brownish Gray & +++ & +++ \\
\hline
\end{tabular}

Remarks : +++ : Very Thick (cover all over PDA sloped ), ++ : Thick (Cover 3/4 PDA sloped on 5 days) 
Tabe.5 Characteristics of Rhizopus spp from Starter of Tempeh Macroscopically

\begin{tabular}{|c|c|c|c|c|c|}
\hline \multirow[t]{2}{*}{ No } & \multirow[t]{2}{*}{ Code Isolate } & \multirow[t]{2}{*}{ Sources of Inoculums } & \multirow[t]{2}{*}{$\begin{array}{l}\text { Color of } \\
\text { Conidia }\end{array}$} & \multicolumn{2}{|c|}{$\begin{array}{c}\text { Observation in Mediaum } \\
\text { Agar }\end{array}$} \\
\hline & & & & Miselia & konidia \\
\hline 1 & RP1 & Ragi Reprima & Gray & +++ & +++ \\
\hline 2 & RP2 & Ragi Reprima & Gray & +++ & +++ \\
\hline 3 & RG1 & Ragi Pemogan & Gray & +++ & +++ \\
\hline 4 & RG2 & Ragi Pemogan & Brownish Gray & +++ & +++ \\
\hline 5 & RI 1 & Ragi perusahan di Seririt & Brownish Gray & +++ & +++ \\
\hline 6 & RI 2 & Ragi perusahan di Seririt & Brownish Gray & +++ & +++ \\
\hline 7 & RA1 & Ragi Perusahan di Pulau Saelus & Gray & +++ & +++ \\
\hline 8 & RA2 & Ragi Perusahan di Pulau Saelus & Brownish Gray & +++ & +++ \\
\hline 9 & RR1 & Ragi tanpa Merk (Singaraja) & Brownish Gray & +++ & +++ \\
\hline 10 & RR2 & Ragi tanpa Merk (Singaraja) & Brownish Gray & +++ & +++ \\
\hline 11 & RL1 & Padangsambian & Brownish Gray & +++ & +++ \\
\hline 12 & RL2 & Padangsambian & Brownish Gray & +++ & +++ \\
\hline 13 & RT1 & Ragi tanpa merk (Sesetan) & Gray & +++ & +++ \\
\hline 14 & RT2 & Ragi tanpa merk (sesetan) & Brownish Gray & +++ & +++ \\
\hline 15 & RF1 & Ragi tanpa merk (Ubung) & Gray & +++ & +++ \\
\hline 16 & RF2 & Ragi tanpa merk (Ubung) & Brownish Gray & +++ & +++ \\
\hline 17 & $\mathrm{RC} 2$ & Ragi tanpa merk (Sesetan) & Brownish Gray & +++ & +++ \\
\hline 18 & $\mathrm{RC} 3$ & Ragi tanpa merk (Sesetan) & Gray & +++ & +++ \\
\hline
\end{tabular}

Remarks : +++ : Very Thick (cover all over PDA sloped ), ++ : Thick (Cover 3/4 PDA sloped on 5 days) 
Tabel.6 Observation macroscopic and Microscopes 10 isolate R.oligosporus from several inoculums

\begin{tabular}{|c|c|c|c|c|c|c|c|c|c|c|c|}
\hline \multirow[t]{2}{*}{ Profile } & \multicolumn{10}{|c|}{ Code Isolate } & \multirow{2}{*}{$\begin{array}{c}\text { R. } \\
\text { oligosporus }\end{array}$} \\
\hline & WR & JT & TT & $\mathrm{TL}$ & $\mathrm{TA}$ & TF & TM & $\mathrm{TP}$ & $\mathrm{TC}$ & TG & \\
\hline Color mycelia & white & white & white & white & white & white & white & White & white & white & White* \\
\hline Color Conidia & $\begin{array}{l}\text { Brownish } \\
\text { Gray }\end{array}$ & $\begin{array}{l}\text { Brownish } \\
\text { Gray }\end{array}$ & $\begin{array}{l}\text { Brownish } \\
\text { Gray }\end{array}$ & $\begin{array}{l}\text { Brownish } \\
\text { Gray }\end{array}$ & $\begin{array}{l}\text { Brownish } \\
\text { Gray }\end{array}$ & $\begin{array}{l}\text { Brownish } \\
\text { Gray }\end{array}$ & $\begin{array}{c}\text { Brownish } \\
\text { Gray }\end{array}$ & $\begin{array}{l}\text { Brownish } \\
\text { Gray }\end{array}$ & $\begin{array}{c}\text { Brownish } \\
\text { Gray }\end{array}$ & $\begin{array}{l}\text { Brownish } \\
\text { Gray }\end{array}$ & $\begin{array}{l}\text { Brownish } \\
\text { Gray }\end{array}$ \\
\hline Shape Conidia & $\begin{array}{l}\text { Globose- } \\
\text { Elipsoidal } \\
\text { (Oval) }\end{array}$ & $\begin{array}{l}\text { Globose- } \\
\text { Elipsoidal } \\
\text { (Oval) }\end{array}$ & $\begin{array}{l}\text { Elipsoidal } \\
\text { (Oval) }\end{array}$ & $\begin{array}{l}\text { Globose- } \\
\text { Elipsoidal } \\
\text { (Oval) }\end{array}$ & $\begin{array}{l}\text { Globose- } \\
\text { Elipsoidal } \\
\text { (Oval) }\end{array}$ & $\begin{array}{l}\text { Globose- } \\
\text { Elipsoidal } \\
\text { (Oval) }\end{array}$ & $\begin{array}{l}\text { Globose- } \\
\text { Elipsoidal } \\
\text { (Oval) }\end{array}$ & $\begin{array}{l}\text { Globose- } \\
\text { Elipsoidal } \\
\text { (Oval) }\end{array}$ & $\begin{array}{l}\text { Globose- } \\
\text { Elipsoidal } \\
\text { (Oval) }\end{array}$ & $\begin{array}{l}\text { Globose- } \\
\text { Elipsoidal } \\
\text { (Oval) }\end{array}$ & $\begin{array}{l}\text { Globose- } \\
\text { ellipsoidal } \\
\text { (Oval) }\end{array}$ \\
\hline Length Conidia & 11.4 & 14.8 & 10 & 12 & 12 & 14.8 & 11.4 & 11.4 & 10 & 10 & $7-24 \mu \mathrm{m}$ \\
\hline $\begin{array}{l}\text { Length } \\
\text { Sporangiosphore }\end{array}$ & 160 & $150-160$ & 160.5 & 170 & 170 & 170 & 170 & 160 & 170 & 170 & $150-400 \mu \mathrm{m}$ \\
\hline $\begin{array}{l}\text { Length } \\
\text { sporangium }\end{array}$ & 90 & 90 & 90 & 100 & 82.5 & 100 & 100.5 & 100.4 & 80 & 80 & $80-120 \mu \mathrm{m}$ \\
\hline $\begin{array}{l}\text { Texture } \\
\text { Sporangiosphore }\end{array}$ & Smooth & Smooth & Smooth & Smooth & Smooth & Smooth & Smooth & Smooth & Smooth & Smooth & Smooth \\
\hline $\begin{array}{l}\text { Length } \\
\text { Columela }\end{array}$ & 27.1 & 27 & 25 & 25 & 30 & 30 & 25 & 25 & 27 & 27 & $25-27 \mu \mathrm{m} *$ \\
\hline Shape Columela & $\begin{array}{l}\text { Globose- } \\
\text { sub } \\
\text { globose }\end{array}$ & $\begin{array}{l}\text { Globose- } \\
\text { sub } \\
\text { globose }\end{array}$ & $\begin{array}{l}\text { Globose- } \\
\text { sub } \\
\text { globose }\end{array}$ & $\begin{array}{l}\text { Globose- } \\
\text { sub } \\
\text { globose }\end{array}$ & $\begin{array}{l}\text { Globose- } \\
\text { sub } \\
\text { globose }\end{array}$ & $\begin{array}{l}\text { Globose- } \\
\text { sub } \\
\text { globose }\end{array}$ & $\begin{array}{l}\text { Globose- } \\
\text { sub } \\
\text { globose }\end{array}$ & $\begin{array}{l}\text { Globose- } \\
\text { sub } \\
\text { globose }\end{array}$ & $\begin{array}{l}\text { Globose- } \\
\text { sub } \\
\text { globose }\end{array}$ & $\begin{array}{l}\text { Globose- } \\
\text { sub } \\
\text { globose }\end{array}$ & $\begin{array}{l}\text { sub globose- } \\
\text { Globose }\end{array}$ \\
\hline Chlamidosphore & $\begin{array}{l}\text { Single- } \\
\text { Short } \\
\text { Chain }\end{array}$ & $\begin{array}{l}\text { Single- } \\
\text { Short } \\
\text { Chain }\end{array}$ & $\begin{array}{l}\text { Single- } \\
\text { Short } \\
\text { Chain }\end{array}$ & $\begin{array}{l}\text { Single- } \\
\text { Short } \\
\text { Chain }\end{array}$ & $\begin{array}{l}\text { Single- } \\
\text { Short } \\
\text { Chain }\end{array}$ & $\begin{array}{l}\text { Single- } \\
\text { Short } \\
\text { Chain }\end{array}$ & $\begin{array}{l}\text { Single- } \\
\text { Short } \\
\text { Chain }\end{array}$ & $\begin{array}{l}\text { Single- } \\
\text { Short } \\
\text { Chain }\end{array}$ & $\begin{array}{l}\text { Single- } \\
\text { Short } \\
\text { Chain }\end{array}$ & $\begin{array}{l}\text { Single- } \\
\text { Short } \\
\text { Chain }\end{array}$ & $\begin{array}{l}\text { Abundant, } \\
\text { single or } \\
\text { Short Chain } \\
* *\end{array}$ \\
\hline
\end{tabular}

* Samson et al., 2005; ** Jennessen et al., 2008 
Tabel.7 Observation macroscopic and Microscopes 10 isolate R.oligosporus from several inoculums

\begin{tabular}{|c|c|c|c|c|c|c|c|c|c|c|}
\hline \multirow[t]{2}{*}{ Profile } & \multicolumn{9}{|c|}{ Code Isolate } & \multirow[t]{2}{*}{ R. oligosporus } \\
\hline & TS & $\mathrm{RG}$ & RI & RA & $\mathrm{RR}$ & $\mathrm{RL}$ & RT & $\mathrm{RF}$ & $\mathrm{RC}$ & \\
\hline Color mycelia & white & white & white & white & white & white & white & white & white & White* \\
\hline Color konidia & $\begin{array}{l}\text { Brownish } \\
\text { Gray }\end{array}$ & $\begin{array}{l}\text { Brownish } \\
\text { Gray }\end{array}$ & $\begin{array}{l}\text { Brownish } \\
\text { Gray }\end{array}$ & $\begin{array}{l}\text { Brownish } \\
\text { Gray }\end{array}$ & $\begin{array}{l}\text { Brownish } \\
\text { Gray }\end{array}$ & $\begin{array}{l}\text { Brownish } \\
\text { Gray }\end{array}$ & $\begin{array}{l}\text { Brownish } \\
\text { Gray }\end{array}$ & $\begin{array}{l}\text { Brownish } \\
\text { Gray }\end{array}$ & $\begin{array}{l}\text { Brownish } \\
\text { Gray }\end{array}$ & Brownish Gray \\
\hline Shape Conidia & $\begin{array}{l}\text { Globose- } \\
\text { Elipsoidal } \\
\text { (Oval) }\end{array}$ & $\begin{array}{l}\text { Globose- } \\
\text { Elipsoidal } \\
\text { (Oval) }\end{array}$ & $\begin{array}{l}\text { Globose- } \\
\text { Elipsoidal } \\
\text { (Oval) }\end{array}$ & $\begin{array}{l}\text { Elipsoidal } \\
\text { (Oval) }\end{array}$ & $\begin{array}{l}\text { Elipsoidal } \\
\text { (Oval) }\end{array}$ & $\begin{array}{l}\text { Elipsoidal } \\
\text { (Oval) }\end{array}$ & Globose & $\begin{array}{l}\text { Elipsoidal } \\
\text { (Oval) }\end{array}$ & $\begin{array}{l}\text { Elipsoidal } \\
\text { (Oval) }\end{array}$ & $\begin{array}{l}\text { Globose- } \\
\text { ellipsoidal } \\
\text { (Oval) }\end{array}$ \\
\hline Length Conidia & 10 & 10 & 12 & 12 & 12 & 20 & 12 & 20 & 12 & $7-24 \mu \mathrm{m}$ \\
\hline $\begin{array}{l}\text { Length } \\
\text { Sporangiosphore }\end{array}$ & 170 & 150 & 170 & 170 & 170 & 170.1 & 170.1 & 170 & 170 & $150-400 \mu \mathrm{m}$ \\
\hline $\begin{array}{l}\text { Length } \\
\text { sporangium }\end{array}$ & 80 & 80 & 90 & 90 & 90 & 90.1 & 92 & 85 & 90 & $80-120 \mu \mathrm{m}$ \\
\hline $\begin{array}{l}\text { Tekstur } \\
\text { Sporangiospore }\end{array}$ & Smooth & Smooth & Smooth & Smooth & Smooth & Smooth & Smooth & Smooth & Smooth & Smooth \\
\hline Length kolumela & 27 & 27 & 27.1 & 27.1 & 27.1 & 27.1 & 27.1 & 27.1 & 27.1 & $25-27 \mu \mathrm{m} *$ \\
\hline $\begin{array}{l}\text { Bentuk } \\
\text { Columela }\end{array}$ & $\begin{array}{l}\text { Globose- } \\
\text { sub } \\
\text { globose }\end{array}$ & $\begin{array}{l}\text { Globose- } \\
\text { sub } \\
\text { globose }\end{array}$ & $\begin{array}{l}\text { Globose- } \\
\text { sub } \\
\text { globose }\end{array}$ & $\begin{array}{l}\text { Globose- } \\
\text { sub } \\
\text { globose }\end{array}$ & $\begin{array}{l}\text { Globose- } \\
\text { sub } \\
\text { globose }\end{array}$ & $\begin{array}{l}\text { Globose- } \\
\text { sub } \\
\text { globose }\end{array}$ & $\begin{array}{l}\text { Globose- } \\
\text { sub } \\
\text { globose }\end{array}$ & $\begin{array}{l}\text { Globose- } \\
\text { sub } \\
\text { globose }\end{array}$ & $\begin{array}{l}\text { Globose- } \\
\text { sub } \\
\text { globose }\end{array}$ & $\begin{array}{l}\text { sub globose- } \\
\text { Globose }\end{array}$ \\
\hline Clamidosphore & $\begin{array}{l}\text { Single- } \\
\text { Short } \\
\text { Chain }\end{array}$ & $\begin{array}{l}\text { Single- } \\
\text { Short } \\
\text { Chain }\end{array}$ & $\begin{array}{l}\text { Single- } \\
\text { Short } \\
\text { Chain }\end{array}$ & $\begin{array}{l}\text { Single- } \\
\text { Short } \\
\text { Chain }\end{array}$ & $\begin{array}{l}\text { Single- } \\
\text { Short } \\
\text { Chain }\end{array}$ & $\begin{array}{l}\text { Single- } \\
\text { Short } \\
\text { Chain }\end{array}$ & $\begin{array}{l}\text { Single- } \\
\text { Short } \\
\text { Chain }\end{array}$ & $\begin{array}{l}\text { Single- } \\
\text { Short } \\
\text { Chain }\end{array}$ & $\begin{array}{l}\text { Single- } \\
\text { Short } \\
\text { Chain }\end{array}$ & $\begin{array}{l}\text { Abundant, } \\
\text { single or Short } \\
\text { Chain ** }\end{array}$ \\
\hline
\end{tabular}

* Samson et al., 2005; ** Jennessen et al., 2008 
Fig.1 Color of colony and mycelia Rhizopus spp from Herbicus leaves, Tectona leaves, Tempeh and starter of tempeh Incubation 3 days temetarure $30^{\circ} \mathrm{C}$

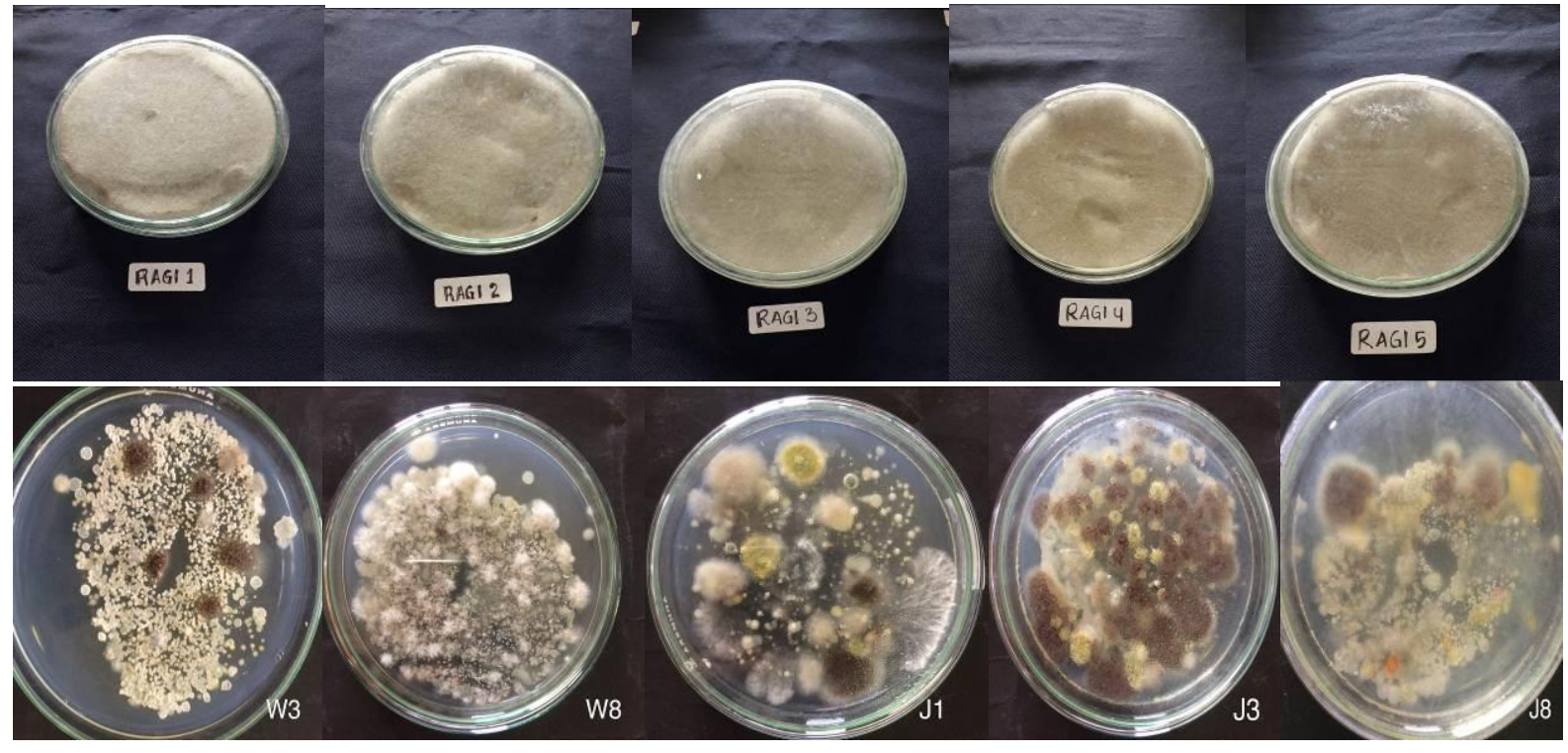

Fig.2 Purification Isolate $R$. oligosporus Incubation 5 days in macroscopically
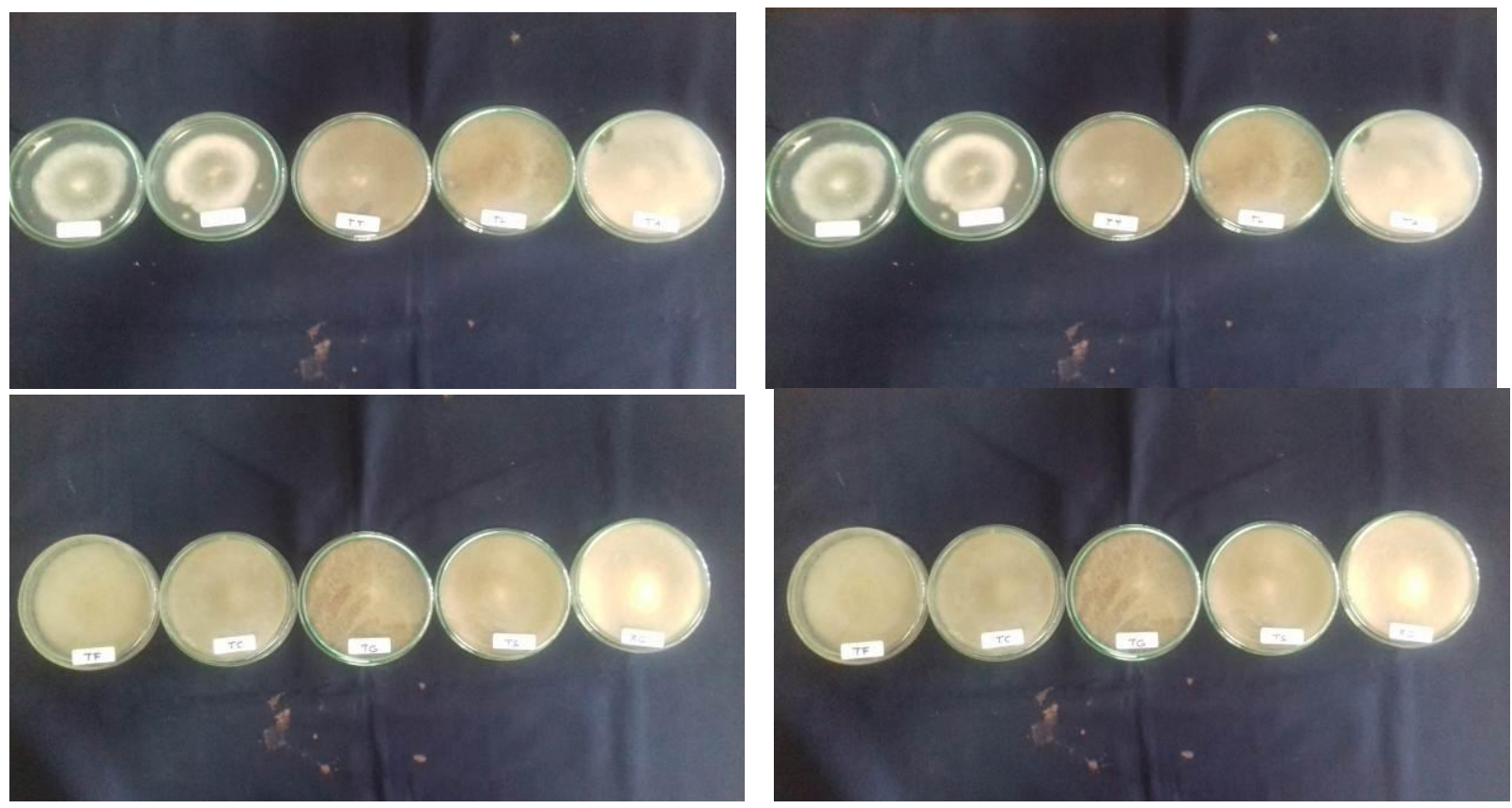
Fig.3 Morphology Rhizopus oligosporus (400 x ) ( $\mathrm{A}=$ Conidia, $\mathrm{B}=$ Sporangiosphore, $\mathrm{C}=$ Conidiophore, $\mathrm{D}=$ Stolon dan $\mathrm{E}=$ Rhizoid

Isolate from Herbicus Leaves

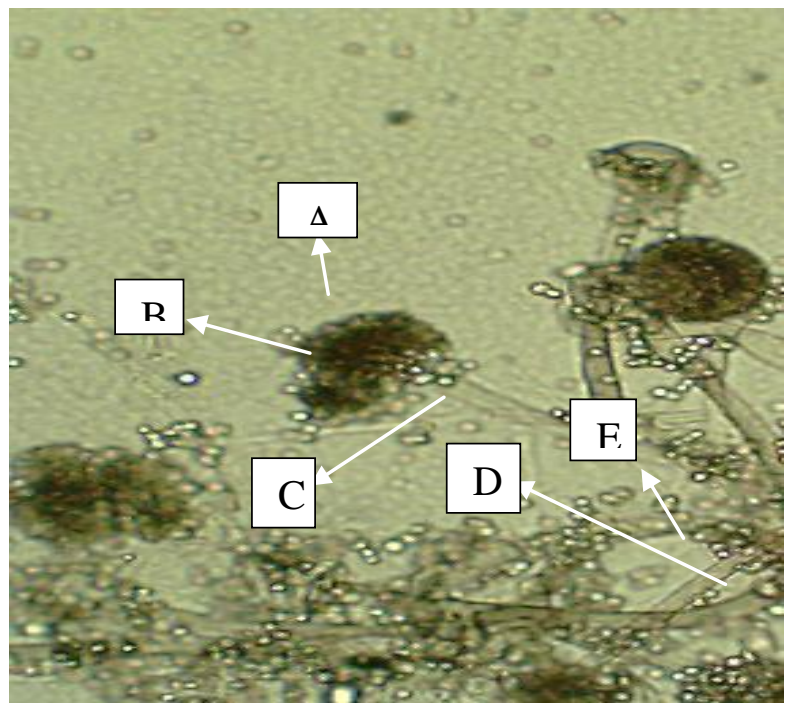

Isolate from Tempeh

\section{Isolate from Tectona leaves}

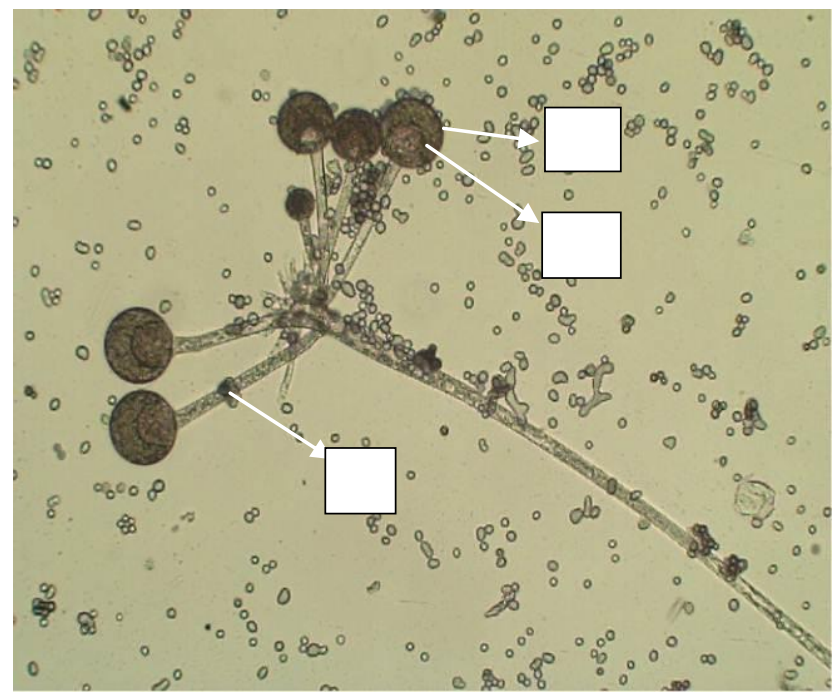

Isolate from Starter of Tempeh
In physiological characterization, the ability of Rhizopus spp. to grow at $33^{\circ} \mathrm{C}, 42^{\circ} \mathrm{C}, 46^{\circ} \mathrm{C}$, $48^{\circ} \mathrm{C}$, and $51^{\circ} \mathrm{C}$ was examined.

The group of fungi that has the most role in making tempe is the genus Rhizopus. Rhizopus sp molds has been known for a long time as a fungus that plays a major role in the process of fermentation of soybeans into tempeh. Rhizopus sp will form a compact white solid called a fine thread / biomass.

Fine yarn / biomass is caused by the fungus mycelia growing on the surface of the soybean seeds and connecting the soybean seeds. Rhizopus sp types are so diverse that they need to be isolated and their morphology and characteristics identified. Identification based on fungal morphology is by observing sporangiophores, sporangium and sporangiospores (Jennessen et al., 2008; Dewi and Aziz 2011; Chang-Tien et al., 2009)). $R$. oligosporus is a fungus that belongs to the class Zygomycetes, which is one of two classes in the phylum Zygomycota. ${ }^{[5]} R$. oligosporus belongs to the Rhizopus microsporus group. This group is made of taxa with similar morphology that are associated with undesired metabolite production, pathogenesis and food fermentation. Although other varieties in $R$. microscopus may be harmful, $R$. oligosporus is not associated with production of potentially harmful metabolites. It is not found in nature and is frequently used by humans. ${ }^{[6]} R$. oligosporus strains have a large (up to $43 \mathrm{~mm}$ ) and irregular spores with the most variable sizes. This is, for instance, reflected as high values in the spore volume (96-223 mm3/spore).R. oligosporus has large, subglobose to globose spores, and high proportion irregular spores $(>10 \%)$. $R$. oligosporusalso has spores with nonparallel valleys and ridges, and plateaus that sometimes are granular (Yanai et al., 1992; Hessel Tine, 1940; Pitt and Hocking, 1985; Samson et al., 2005) 
$R$. oligosporus can grow optimally at a temperature of $30-35{ }^{\circ} \mathrm{C}$, with a minimum temperature of $12^{\circ} \mathrm{C}$, and a maximum temperature of $42{ }^{\circ} \mathrm{C}$. Growth of $\mathrm{R}$. oligosporus has the characteristics of brownish gray colonies with a height of $1 \mathrm{~mm}$ or more. Single sporangiophores or in groups with smooth or slightly rough walls, with a length of more than $1000 \mu \mathrm{m}$ and a diameter of 10-18 $\mu \mathrm{m}$. Sporangia globosa which when brown is black brown in color, with a diameter of 100$180 \mu \mathrm{m}$. Chlamydospores are many, single or short, colorless chains, containing granules, formed on hyphae, sporangiophores and sporangia. The form of klamidospora globosa, ellipse or cylindrical with a size of 7-30 $\mu \mathrm{m}$ or 12-45 $\mu \mathrm{m} \times 7-35 \mu \mathrm{m}$ (Madigan and Martinko, 2006; Pitt and Hocking, 1985; Samson et al., 2005). Table 6 can be seen 10 isolate R.oligosporus from several inoculums

Indonesia in this study belonging to $R$. delemar and $R$. microsporus. Indeed, several members of Rhizopus, such as $R$. oligosporus, $R$. oryzae, $R$. arrhizus, and $R$. stolonifer were previously reported in Indonesia from inoculants of tempeh (ragi) and from fresh tempeh (Dewi and Aziz 2011; Prihatna and Suwanto 2007). In the current systematic of Rhizopus, $R$. arrhizus is treated as a synonym of $R$. oryzae a s proposed by Abe et al., (2010)

A popular Indonesian food, Tempeh, is created by fermenting soybeans in combination with $R$. oligosporus (Dewi and Aziz 2011; Chang-Tien et al., 2009; Prihatna and Suwanto 2007) In order to create tempeh, soybeans must first be soaked in water (usually overnight) at a temperature similar to the environment it is placed in. The soybean's outer covering is then removed and the beans are partially cooked. Lactic acid bacteria, like Lactococcus and Lb. casei species, play a major role in the fermentation of tempeh. ${ }^{[8]}$ For the tempeh to ferment there needs to be a suitable, pure inoculums. Also, spores with a tendency for fast germ inability are needed, as well (Caplice, et al., 1999; Prihatna and Suwanto 2007). In order for the tempeh to attain its characteristic compact 'cake' form after fermentation, the soybeans become compressed due to the mycelia of $R$. oligosporus. ${ }^{[8]}$ Rapidly growing mycelia helps speed up the growth of this fungus. Because mycelia are quite sensitive to dehydration and adverse temperatures, preserving tempeh for extended periods of time can be challenging (Dewi and Aziz 2011; Chang-Tien et al., 2009; Prihatna and Suwanto 2007) When the soybeans are bound together by the white mycelium, the fungus releases enzymes that make this heavily protein-rich product more digestible for humans (Tempeh-like foods can also be created from cereal grains such as wheat and rice. Many times, a good inoculum for this new fermentation actually comes from tiny pieces of old tempeh that have already been fermented (Caplice, et al., 1999; Prihatna and Suwanto 2007; Chang-Tien et al., 2009)

Isolates other than 72 isolates that were successfully isolated (besides the 19 isolates) there were 24 isolates including the genus Rhyzopus (data not supported). All isolates were characterized by nonseptic hyphae, had stolons and rhizoids that were black when was old, sporangiophora growing basically also contained rhizoid, large and black sporangia, larger columella and apophysis, such as jackets, buying sporangia at the time of sporangiofora, growing, and also containing rhizoid, large and black sporangia, larger columella and apophysis, such as a jacket, buying sporangia at the time of sporangiofora, increasing, and forming mycelium like cotton as argued by Fardiaz (1989; Dewi and Aziz (2011); Chang-Tien et al., (2009); Prihatna and Suwanto (2007)). Table 7. Can be seen 9 isolate R.oligosporus from several inoculums $R$. oryzae, $R$. oligosporus and other mold types were found, with populations ranging from 1.5 x 102cfu / g - 9.5x $102 \mathrm{cfu} / \mathrm{g}, 0.5$ x $103-9.6 \mathrm{x}$ 
$103 \mathrm{cfu} / \mathrm{g}$ and $1.6 \times 102 \mathrm{cfu} / \mathrm{g}-8.6 \times 102 \mathrm{cfu} / \mathrm{g}$ A total of 72 samples taken from various sources of inoculums, $R$. oligosporus found as many as 12 from waru leaves, 3 samples from teak leaves, 17 samples from various brands of tempeh and as many as 11 samples from tempe yeast were identified macroscopically based on color conidia with brownish gray color and the growth of mycelia and conidia is very dense. Macroscopically and microscopically identified as many as 19 local isolates of $R$. oligosporus which have the potential as selected fungi in food processing.

\section{Acknowledgment}

The research was supported from the ministry of research and Technology, High Education of Republic of Indonesia for the funding. Herewith, I would like to great thank you for all contributors that supporting moral and material to reach this achievement of this article. Thank you very much also we express to the Board of research and applied of the society of Udayana University.

\section{References}

Abe A, Oda Y, Asano K, Sone T. 2010. A molecular phylogeny based taxonomy of the genus Rhizopus. Biosc Biotechn Biochem $74: 1325 \mathrm{e} 31$. http://dx.doi.org/10.1271/bbb.90718.

Caplice, Elizabeth; Fitzgerald, Gerald F. (1999). "Food Fermentations: role of microorganisms in food production and preservation". International Journal of Food Microbiology. 50: 143. doi:10.1016/S0168-1605(99) 00082-3. Retrieved 27 May 2014

Chang-Tien, Chang; Hsu, Cheng-Kuang; Chou, Su-Tze; Chen, Ya-Chen; Huang, FengSheng; Chung, Yun-Chin (2009). "Effect of fermentation time on the antioxidant activities of tempeh prepared from fermented soybean using Rhizopus oligosporous". International Journal of
Food Science and Technology. 44: 799. doi:10.1111/j.1365-2621.2009.01907.x. Retrieved 27 May 2014

Dewi RS and Aziz S. 2011. Isolasi Rhizopus oligosporus pada beberapa inokulum tempe di Kabupaten Banyumas. Molekul 6:93e104.

Fardiaz S., 1989, Mikrobiologi Pangan, PAUIPB, Bogor

Hessel Tine, W; Swain, E.W.; Wang, Hwa L. (1940). "Mass production of Rhizopus oligosporus spores and their application in tempeh fermentation" (PDF). Journal of Food Science. 40: 168-170. doi:10.1111/j.1365-

2621.1975.tb03762.x. Retrieved 29 May 2014.

Jennessen, J.; Nielsen, K.F.; Houbraken, J.; Lyhne, E.K.; Schnürer, J.; Frisvad, J.C.; Samson, R.A. (2005). "Secondary metabolite and mycotoxin production by the Rhizopus microsporus group". Journal of Agricultural and Food Chemistry. 53: 1833-1840. doi:10.1021/jf048147n. PMID 15740082.

Jennessen, J., J. Schnurer, J. Olsson, R.A. Samson, and J. Dijksterhuis, 2008, Morphological characteristics of sporangiospores of the tempe fungus Rhizopus oligosporus differentiate it from other taxa of the R. microsporus group. Mycol. Res, Vol.112, 547-63.

Kobayasi, Sin-ya; Naoto, OKAZAKI; Takuya, KosEKI (1992). "Purification and Characterization of an Antibiotic Substance Produced from Rhizopus oligosporus IFO 8631". Biosci. Biotechnol. Biochem. 56 (1): 94-98. doi:10.1271/bbb.56.94. PMID 1368137.

Madigan, M.T., and J.M. Martinko, 2006, Brock Biology of Microorganisms 11th ed. Pearson Education, New Jersey.

Pitt, J.I. and A.D Hocking,. 1985. Fungi and Food Spoilage. Academic Press, Australia.

Prihatna C, Suwanto A. 2007. Phenotypic, metabolic, and genetic diversity of the Indonesian strains of Rhizopus 
oligosporous. J Microbiol Indones 1:2732

Rahayu, K. 1988. Bahan Pengajaran Mikrobiologi Pangan PAU Pangan dan Gizi.UGM, Yogyakarta.

Samson, R.A., E.S. Hoekstra, J.C. Frisvad and O. Filtenborg. 1995. Introduction to Food-Borne Fungi. Baarn and Lyngby, Netherlands

Shurtleff, W. \& Aoyagi, A. 2001. The book of tempeh. 2 2. Ten Speed Press. Berkeley, California pp.

Nout, M.J.R. (1989). "Effect of Rhizopus and Neurospora spp. on growth of Aspergillus flavus and A. parasiticus and accumulation of aflatoxin BI in groundnut". Mycological Research. 93: 518-523. doi:10.1016/s09537562(89)80046-2.

Yanai, K; Takaya, N; Kojima, N; Horiuchi, H; Ohta, A; Takagi, M (1992). "Purification of two chitinases from Rhizopus oligosporus and isolation and sequencing of the encoding genes". American Society for Microbiology: 7398. Retrieved 29 May 2014.

Zheng RY, Chen GQ, Huang H, Liu XY. 2007. A monograph of rhizopus. Sydowia 59:273-372

\section{How to cite this article:}

Duniaji, A. S., W. Wisaniyasa, N. N. Puspawati and dan N. M. Indri H. 2019. Isolation and Identification of Rhizopus Oligosporus Local Isolate Derived from Several Inoculum Sources. Int.J.Curr.Microbiol.App.Sci. 8(09): 1085-1098. doi: https://doi.org/10.20546/ijcmas.2019.809.126 